\title{
Review: mothers with postpartum depression had to readjust expectations, cope with loss, and find ways to meet needs
}

\author{
Beck CT. Postpartum depression: a metasynthesis. Qual Health Res 2002 Apr;12:453-72.
}

\section{QUESTION: What are the experiences of mothers who have postpartum depression?}

\author{
Data sources \\ Studies were identified by searching CINAHL, PsycLIT, \\ Index Medicus, Social Sciences Citation Index, Ameri- \\ can Humanities Index, Anthropological Literature, \\ Sociological Abstracts, and Dissertation Abstracts.
}

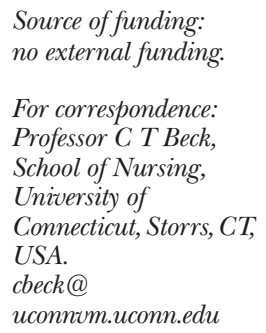

\section{Study selection}

Studies were selected if they were qualitative studies on postpartum depression.

\section{Data extraction}

Data were extracted on the key metaphors in each study and their relations with each other. Reciprocal translations were done so that the metaphors within

\section{COMMENTARY}

Postpartum depression is a substantial health problem affecting $12-16 \%$ of women. The meta-synthesis of 18 qualitative studies by Beck provides insight into the experiences of women living with this illness. Much of the existing research literature focuses on issues such as prevalence, risk factors, identification, management, and effects. This qualitative study adds another important dimension to our understanding of postpartum depression.

The 4 overarching themes that emerged from the meta-synthesis capture the range of feelings, emotions, fears, conflicts, and challenges commonly experienced by women with postpartum depression. Because this illness is underdiagnosed and undertreated, guidelines for clinical practice recommend that health professionals attend to complaints of emotional distress and assess the mother's mood at postnatal follow up visits. ${ }^{2}$ The spiralling downward theme from Beck's meta-synthesis brings to light the fact that postpartum depression is characterised by an array of emotions, including anxiety, guilt, and being overwhelmed. These feelings should not be discounted as "normal" because this could contribute to silencing and social withdrawal; rather, such feelings should be explored as possible symptoms of a serious illness that affects the mothers and their families.

Treatment for postpartum depression can include antidepressant medication as well as cognitive behavioural therapy, interpersonal psychotherapy, education, and support. ${ }^{3}$ The results of the meta-synthesis have implications for non-pharmacological intervention. The importance of addressing the myths of motherhood and unrealistic societal expectations is highlighted. Clinicians should provide opportunities to discuss the realities of parenting and reassure women that they are not "incompetent" or "bad mothers." 4 Another noteworthy finding is that mothers with postpartum depression experience a pervasive sense of loss, with their losses taking many forms. As pointed out by Beck, loss and grief frameworks can be useful to clinicians in designing interventions.

Through understanding the lived experience of postpartum depression, healthcare providers are better equipped to identify depression in women during the postnatal period as well as to intervene appropriately in response to each woman's unique experience and individual needs.

Wendy Sword, RN, PhD McMaster University Hamilton, Ontario, Canada

1 Marcus SM, Barry KL, Flynn HA, et al. Treatment guidelines for depression in pregnancy. Int J Gynecol Obstet 2001;72:61-70.

2 Stowe ZN, Nemeroff CB. Women at risk for postpartum-onset major depression. Am J Obstet Gynecol 1995;175:639-45.

Miller LJ. Postpartum depression. JAMA 2002;287:762-5.

4 Gibson J. Motherhood: unrealistic expectations? Practising Midwife 2001;4:32-4. each study could be related to those of other studies. The translations were synthesised by comparing them and determining the encompassing metaphors and themes.

\section{Main findings}

18 studies ( 2 unpublished) were included. Study settings were USA (6 studies), UK (8 studies), Australia (3 studies), and Canada (1 study). Study designs included phenomenology (6 studies), general descriptive design (4 studies), ethnography (2 studies), grounded theory (2 studies), and 1 case study. The themes reflected 4 perspectives related to postpartum depression. (1) Incongruity between expectations and reality of motherhood: personal and societal beliefs about how mothering leads to complete fulfilment and happiness resulted in unrealistic expectations. Women felt that no other mothers shared their negative reactions to childbirth, and they perceived themselves to be bad mothers. (2) Spiralling downward: postpartum depression led to many distressing feelings such as anxiety, being overwhelmed, obsessive thinking, anger, cognitive impairment, isolation/loneliness, guilt, and contemplation of harming herself or her infant. (3) Pervasive sense of loss: mothers experienced loss of control, including control of their thought processes and emotions; loss of autonomy and time, which contributed to a loss of physical appearance; loss of self, both of who they are and their former selves; loss of relationships with partners, children, and family/friends; and loss of voice (from fear of what might happen if they voiced their feelings or fear of burdening others). (4) Making gains: recovery required surrendering. Mothers had to admit that they needed help and be willing to seek help even though they felt ashamed, embarrassed, and afraid of being labelled a bad mother or failure. Struggling to survive: once women admitted they needed help, they struggled to find appropriate treatment. Some women turned to spirituality. Other women attended support groups and found hope through realising that they were not alone. Reintegration and change: mothers learnt to adjust their unrealistic expectations and regain control by recognising and finding ways of meeting their own needs. Recovery involved acceptance or resolution of their conflicts, and mothers felt a sense of change after the losses they experienced with postpartum depression.

\section{Conclusions}

Mothers with postpartum depression had to readjust unrealistic expectations about becoming a mother; reconcile with losses of control, self, relationships, and voice; and find ways of reintegrating by recognising their own needs and getting those needs met. 